\title{
Detecting Primary Transmitters via Cooperation and Memory in Cognitive Radio
}

\author{
A. Betran-Martinez, O. Simeone and Y. Bar-Ness \\ CWCSPR NJIT, Newark, New Jersey 07102-1982, USA \\ \{osvaldo.simeone, yeheskel.barness\}@ njit.edu
}

\begin{abstract}
Effective detection of the activity of primary transmitters is known to be one of the major challenges to the implementation of cognitive radio systems. In this paper, we investigate the use of cooperation and memory (using tools from change detection) as means to enhace primary detection. We focus on the simple case of two secondary users and one primary source. Numerical results show the relevant performance benefits of both cooperation and memory.
\end{abstract}

\section{INTRODUCTION AND MOTIVATION}

Cognitive Radio builds on the software-defined radio technology, and refers to a smart wireless comunication system that is able to learn from its environment and adapt its conditions of work towards given quality of service requirements. Cognitive radio improves the utilization of the radio spectrum by allowing the co-existence of primary (licensed) users and secondary (unlicensed) users on the same bandwith [1]. Primary users have preferential access to the bandwith whereas secondary users are required to vacate the spectrum as soon as they detect primary activity so as to minimize interference.

In this paper, we focus on a simple scenario with one primary transmitter $(\mathrm{P})$, and two secondary $\left(\mathrm{U}_{1}\right.$ and $\left.\mathrm{U}_{2}\right)$ transmitters communicating with a secondary receiver over Rayleigh fading channels as illustrated in Fig.1-(a). Our main goal is to study the interplay and relative merits of two techniques aimed at minimizing the primary activity detection time. One approach consists in exploiting cooperation between the two secondary users, whereby one of the users acts as an amplify-and-forward relay for the other, as proposed in [2]. The other approach is leveraging memory using tools from change detection [3], which has so far received little attention in the field of cognitive radio, e.g., [1].

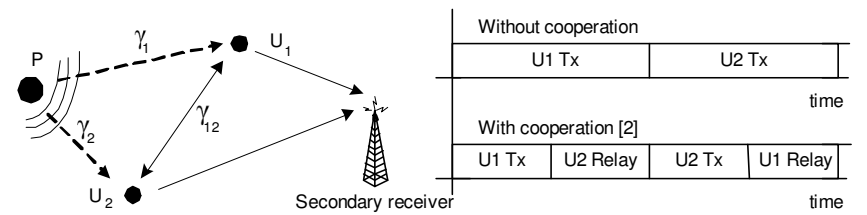

(a)

(b)

Fig. 1. a) System model. b) Slotted transmission protocol.

\section{Contributions}

In this paper, we study the interplay between cooperation and memory in primary activity detection. The cooperative scheme proposed in [2] works as illustrated in Fig. 1-(b): for each pair of slots, secondary user $\mathrm{U}_{i}(i=1,2)$ transmits in the first slot, whereas the other user $\mathrm{U}_{j}(j \neq i)$ amplifies and forwards the received signal in the second slot. Whenever not transmitting, secondary users $U_{1}$ and $U_{2}$ sense the channel in order to detect the activity of the primary. Cooperation is generally proved to be helpful if the average channel power received by the two users $U_{1}$ and $U_{2}$ from the primary is unbalanced (i. e., $\gamma_{i}>\gamma_{j}$ with $i \neq j$, see Fig. 1-(a)) [2].
Besides cooperation, a second technique that is expected to provide enhanced primary detection capability is change detection [3], that exploits memory in the detection process. In this framework, the optimal algorithm is Cumulative Sum (CUSUM), which is defined by a recursive decision function $g_{k}=\left(g_{k-1}+s_{k}\right)^{+}$, where $s_{k}$ is the log-likelihood ratio calculated in each slot. The stopping rule is $t_{a}=\min \left\{k: g_{k} \geq h\right\}$ with $t_{a}$ being the alarm time (i.e., when primary is detected) and $h$ is a chosen threshold. Log-likelihood ratios are evaluated at the secondary nodes by employing a Gaussian approximation for the received signal and assuming knowledge of the average channels $\gamma_{1}, \gamma_{2}, \gamma_{12}$ in Fig. 1-(a).

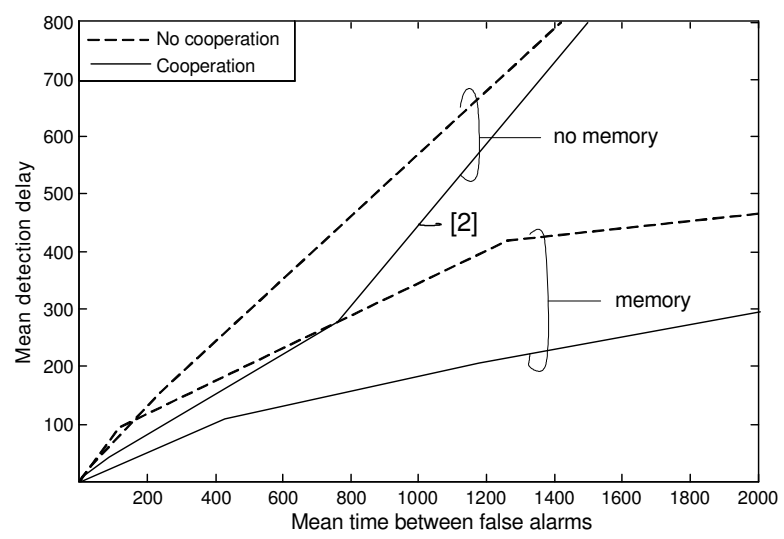

Fig. 2. System performance $\left(\gamma_{1}=0 d B, \gamma_{2}=-10 d B, \gamma_{12}=-3 d B\right)$.

\section{RESULTS}

Fig. 2 exemplifies our numerical results on the performance of cooperation [2], memory (change detection) and a combination thereof, in terms of mean detection delay (i.e., the average number of slots needed for the two secondary users to detect an active primary transmitter) versus mean time between false alarms [3]. Our findings confirm that the benefits of cooperation depend on the system topology (and in particular, on the unbalance between $\gamma_{1}$ and $\gamma_{2}$ ), whereas change detection is particularly effective at low SNR [3]. Moreover, the performance advantage of a joint application of both approaches is remarkable. As future work, we are currently pursuing an analytical study of system performance based on the results in [3].

\section{REFERENCES}

[1] S. Haykin, "Cognitive Radio: Brain-Empowered Wireless Communications", IEEE J. Select. Areas Commun., vol. 23, no. 2, pp. 201-220, Feb. 2005.

[2] G. Ganesan and Y. Li, "Agility Improvement through Cooperative Diversity in Cognitive Radio", in Proc. IEEE GLOBECOM '05, Nov. 2005.

[3] M. Basseville and I. Nikiforov, Detection of Abrupt Changes: Theory and Application, Prentice Hall, 1993. 